\title{
ON THE PLANNER'S LOSS DUE TO LACK OF INFORMATION IN BAYESIAN MECHANISM DESIGN
}

\author{
JOSÉ R. CORREA* AND NICOLÁS FIGUEROA** \\ * School of Business, Universidad Adolfo Ibáñez, Av Diagonal las Torres 2640, Peñalolén, Santiago, Chile; \\ http://jcorrea.uai.cl \\ ** Centro de Economía Aplicada, DII, Universidad de Chile, República 701, Santiago, Chile; \\ nicolasf@dii.uchile.cl
}

\begin{abstract}
In this paper we study a large class of resource allocation problems with an important complication, the utilization cost of a given resource is private information of a profit maximizing agent. After reviewing the characterization of the optimal bayesian mechanism, we study the informational cost introduced by the presence of private information. Our main result is to provide an upper bound for the ratio between the cost under asymmetric information and the cost of a fully informed designer, which is independent of the combinatorial nature of the problem and tight. We also show that this bound holds for a variation of the Vickrey-Clark-Groves mechanism. Finally we point out implementation issues of the optimal mechanism.
\end{abstract}

\section{INTRODUCTION}

A wide class of problems of the form $\min \left\{c^{t} x \mid x \in \Gamma\right\}$ have been analyzed in the literature and their application to real world problems are innumerable. In this paper we consider such a class of problems with an important and realistic complication, the utilization cost of a given resource $x_{i}$ is private information of a profit maximizing agent .

For example, let us consider a natural situation in supply chain management. A large company needs to procure quantities $D_{i}$ of a given good for its various locations $t_{1}, \ldots, t_{k}$. The good is produced at various locations $s_{1} \ldots, s_{l}$, each of them with a maximum production capacity $Q_{j}$. The delivery of the goods is done through a transportation network in which each link has a cost that is publicly known. This problem, when the production facilities are owned by the company, reduces to a standard minimum cost flow through a network. If, however, the production facilities are owned by private contractors, whose production cost is private information, there is an added layer of difficulty to the problem. Now the company must design a mechanism to minimize expected procurement cost, subject to the feasibility constraints on the network, and inducing contractors to reveal their costs in exchange of a profit.

Date: June 2008.

Key words and phrases. Mechanism Design, Information Cost.

JEL Classification: C60, C72, D44. 
In this paper, we quickly review the characterization of the optimal bayesian (i.e., utilization costs are random variables) mechanism for the whole class of problems with a linear cost function and a fixed constraint set. Then, we analyze two basic problems.

First, we study the informational cost introduced by the presence of private information. Such a consideration is important, because it lies at the heart of an old economic question: To make or to buy? If this cost is small, the organizational cost of acquiring small producers may be high and not worth it. If big, such an acquisition may turn out to be profitable for the company trying to procure goods or services. Our main result is to provide an upper bound for the ratio between the cost under asymmetric information and the cost of a fully informed designer. Specifically, this bound states that for a large class of distributions, which contains those that are symmetric and unimodal, the expected cost of an optimal mechanism is at most twice the cost of an optimal solution obtained by a fully informed planner. Neither this bound nor its tightness depend on the combinatorial nature of the problem, but only on the statistical distribution of private information. The latter bound holds for a variation of the Vickrey-Clark-Groves mechanism as well, and becomes significantly better in some special situations.

On the other hand, we study the computational cost of calculating such a mechanism. We show that such a problem is equivalent to performing parametric linear programming over the set $\Gamma$, which is in general of exponential complexity, even if optimization over $\Gamma$ is simple. For the important class of problems where the set $\Gamma$ is a $0-1$ polytope, however, we give a simple algorithm with the same complexity of the original optimization problem with complete information. For the other problems, we point out that a simple sampling technique which takes advantage of the owners' risk neutrality, gives a random mechanism which yield the same total expected cost as the deterministic one.

\section{The ModeL}

2.1. The Environment. We consider a setting in which scarce resources must be allocated in order to minimize the cost of a given project. The cost of each resource may be public or private information. The planner's goal is to minimize the expected cost of the project. To this end, she can design a mechanism where the owners with private information have incentives to reveal their private information.

In our framework each resource $a \in A$ is represented by a variable $x_{a}$, and is associated with a marginal cost of utilization $c_{a}$. The set $A$ is partitioned into two sets $A_{1}$ and $A_{2}$. Costs of resources $a \in A_{1}$ are private, and thus $c_{a}$ is private information and is distributed according to $F_{a}$, whose bounded support is the interval $\left[\underline{c}_{a}, \bar{c}_{a}\right] \subset \mathbb{R}_{+}$. The distribution $F_{a}$ is assumed to have a density $f_{a}$ which is continuous and strictly positive in $\left[\underline{c}_{a}, \bar{c}_{a}\right]$. For simplicity we also assume that $F_{a}\left(c_{a}\right) / f_{a}\left(c_{a}\right)$ is nondecreasing (satisfied among others by the family of logconcave distributions). Costs of resources $a \in A_{2}$ are public information and equal $c_{a}$. Resources are scarce and subject to

an exogenous feasibility constraint $x \in \Gamma \subseteq \mathbb{R}^{|A|}$, which we assume compact. Therefore, if all costs $c_{a}, a \in A$ were known, the planner would solve $\min \left\{c^{T} x: x \in \Gamma\right\}$. However, costs of resources in 
$A_{1}$ are unknown and thus the planner must design a mechanism to elicit this information in order to minimize expected cost.

We now give a key property that holds in this environment. It states that if the cost of a resource increases, the value of the corresponding variable, in a cost-minimizing solution, does not increase. This intuitive and simple result turns out to be critical for characterizing the optimal mechanism.

Lemma 1. Let $x(c)=\left\{x_{a}(c)\right\}_{a \in A}$ be the minimum cost assignment in $\Gamma$ for a cost vector $c$. Then $x_{a}\left(\cdot, c_{-a}\right)$ is non-increasing for all $a \in A$.

Proof. Consider a cost vector $c$ and let $c^{\prime}$ be defined as $c_{e}^{\prime}=c_{e}$ for all $e \in A-\{a\}$ and $c_{a}^{\prime}=c_{a}+\varepsilon$ for some $\varepsilon>0$. From the definition of $x(c)$ we have that: $c^{T} x(c) \leq c^{T} x\left(c^{\prime}\right)$ and $c^{\prime T} x\left(c^{\prime}\right) \leq c^{\prime T} x(c)$. Summing both terms we obtain $\left(c^{T}-c^{\prime T}\right)\left[x(c)-x\left(c^{\prime}\right)\right] \leq 0$ which is equivalent to $x_{a}(c) \leq x_{a}\left(c^{\prime}\right)$.

As usual, if $x \in \mathbb{R}^{n}, x_{-i}$ denotes the vector in which the $i$-th component is removed. We also define:

$$
f(c)=\prod_{a \in A_{1}} f_{a}(c), \quad f_{-a}(c)=\prod_{e \in A_{1}-\{a\}} f_{a}(c), \quad C=\prod_{a \in A_{1}}\left[\underline{c}_{a}, \bar{c}_{a}\right], \quad C_{-a}=\prod_{e \in A_{1}-\{a\}}\left[\underline{c}_{a}, \bar{c}_{a}\right] .
$$

2.2. Mechanisms. In order to minimize its expected cost, the planner designs a mechanism. In other words, the planner chooses a message space $M_{a}$ for each $a \in A_{1}$, together with assignment and payment rules. Given messages from resource owners, these rules determine the amount of each resource used by the planner and the payment received by each owner. Due to the revelation principle it is enough to set $M_{a}=\left[\underline{c}_{a}, \bar{c}_{a}\right]$ and consider truthful mechanisms.

Therefore, a mechanism is given by assignment rules $\left\{x_{a}\right\}_{a \in A}$, indicating how much of resource $a$ will be used, and a family of payment rules $\left\{t_{a}\right\}_{a \in A_{1}}$, indicating the total payment to the owner of resource $a \in A_{1}$. Naturally, these values depend on the cost revelations of each owner, therefore $x_{a}: C \longrightarrow \mathbb{R}$ and $t_{a}: C \longrightarrow \mathbb{R}$. Our framework allows the payment received by the owner of resource $a$, given revelations $c$, to be random. If this is the case, $t_{a}$ denotes the total expected payment to the owner of resource $a \in A_{1}$.

The payoff of the owner of resource $a$, with $\operatorname{cost} c_{a}$, when reporting a cost $c_{a}^{\prime}$ is given by:

$$
U_{a}\left(c_{a}, c_{a}^{\prime}\right)=\int_{C_{-a}}\left[t_{a}\left(c_{a}^{\prime}, c_{-a}\right)-c_{a} x_{a}\left(c_{a}^{\prime}, c_{-a}\right)\right] f_{-a}\left(c_{-a}\right) d c_{-a} .
$$

The payoff of a resource owner with cost $c_{a}$ is then:

$$
V_{a}\left(c_{a}\right)=\max _{c_{a}^{\prime} \in C_{a}} U_{a}\left(c_{a}, c_{a}^{\prime}\right)
$$

We must also consider mechanisms that give a positive utility to owners and satisfy the feasibility constraints. We can thus give the following definition.

Definition 2. A mechanism $(x, t) \equiv\left(\left\{x_{a}\right\}_{a \in A},\left\{t_{a}\right\}_{a \in A_{1}}\right)$ is feasible if and only if for all cost realizations $c$ the following hold: 


$$
\begin{aligned}
& \text { (IC) } \quad V_{a}\left(c_{a}\right)=U_{a}\left(c_{a}, c_{a}\right) \quad \text { for all } a \in A_{1} \text {, } \\
& \text { (PC) } V_{a}\left(c_{a}\right) \geq 0 \quad \text { for all } a \in A_{1} \text {, } \\
& \text { (F) } \quad x(c) \in \Gamma
\end{aligned}
$$

With this, we can write the problem of a cost-minimizing designer as

$$
\min \left\{\int_{c \in C}\left(\sum_{a \in A_{1}} t_{a}(c)+\sum_{a \in A_{2}} c_{a} x_{a}(c)\right) f(c) d c:(x, t) \text { is feasible }\right\} .
$$

2.3. The Optimal Bayesian Mechanism. Using by now standard arguments introduced by Myerson [6] and extended among others by Elkind et. al. [2] (see [7, Chapter 13] for a detailed treatment) we can characterize the optimal Bayesian mechanism relying on Lemma 1. For completeness we have included a proof in Appendix A. Indeed, the optimal mechanism can be written as the solution to the following control problem

$$
\begin{gathered}
\min _{\left\{x_{a}(c)\right\}_{a} \in A} \int_{c \in C}\left(\sum_{a \in A_{1}} x_{a}(c)\left[c_{a}+\frac{F_{a}\left(c_{a}\right)}{f_{a}\left(c_{a}\right)}\right]+\sum_{a \in A_{2}} x_{a}(c) c_{a}\right) f(c) d c \\
\text { s.t. } \quad x(c) \in \Gamma \text { and } \nu_{a}\left(c_{a}\right) \text { non-increasing for all } a \in A_{1} .
\end{gathered}
$$

Here, $\nu_{a}\left(c_{a}\right):=\int_{C_{-a}} x_{a}\left(c_{a}, c_{-a}\right) f_{-a}\left(c_{-a}\right) d c_{-a}$ is the expected utilization of resource $a$ for $a \in A_{1}$.

Because of Lemma 1 , and the assumption that $F_{a}\left(c_{a}\right) / f_{a}\left(c_{a}\right)$ is increasing, we can relax the constraint asking for $\nu_{a}\left(c_{a}\right)$ non-increasing and solve the above problem pointwise to obtain a feasible solution. Therefore, we can characterize the optimal mechanism.

Proposition 3. The optimal assignment rules $\bar{x}(c)=\left\{\bar{x}_{a}(c)\right\}_{a \in A}$ are those solving, for each cost revelations $\left\{c_{a}\right\}_{a \in A_{1}}$, the following optimization problem:

$$
\min _{y \in \Gamma} \sum_{a \in A_{1}}\left(c_{a}+\frac{F_{a}\left(c_{a}\right)}{f_{a}\left(c_{a}\right)}\right) y_{a}+\sum_{a \in A_{2}} c_{a} y_{a},
$$

and an optimal payment rule is given by

$$
\bar{t}_{a}(c)=c_{a} \bar{x}_{a}(c)+\int_{c_{a}}^{\bar{c}_{a}} \bar{x}_{a}\left(t, c_{-a}\right) d t .
$$

In other words, $x(c)$ is the minimum cost assignment in $\Gamma$ with virtual costs $c_{a}^{\prime}=c_{a}+F_{a}\left(c_{a}\right) / f_{a}\left(c_{a}\right)$ for all $a \in A_{1}$, and $c_{a}^{\prime}=c_{a}$ for all $a \in A_{2}$.

\section{LOSS DUE TO LACK OF INFORMATION}

The presence of private information among resource owners increases the cost of performing a given task. A natural problem, with relevant practical implications, is to quantify the relationship between the cost under complete and incomplete information. The former corresponds to a situation where the planner owns the different resources and the technology needed for their production, 
therefore knowing exactly the production costs. The latter corresponds to a decentralized situation, where the planner has outsourced the production of necessary inputs, and therefore does not know precisely their production costs. Since outsourcing can imply important savings in terms of managerial effort, it is critical to know how much is a firm losing by spinning off some of its components, or how much is a central planner losing by privatizing some key components of a planned economy. Moreover, with incomplete information, a cost-minimizing planner does not necessarily assigns resources efficiently (since he considers modified costs), so we consider the question of the expected cost of an efficient mechanism, and its comparison to the cost-minimizing and the full information ones.

Interestingly, both comparisons can be done independently of the combinatorial structure of the problem (given by the set $\Gamma$ ), and depend on the nature of the incomplete information (given by the distribution functions $F_{a}$ ). The critical lemma is the following:

Lemma 4. If the distribution $F$ with density $f$ satisfies that $\mathbb{E}(X \mid X \leq y) \geq y / \alpha$, where $X$ is drawn according to $F$, then for $[a, b] \subset \mathbb{R}_{+}$and $g(\cdot)$ a non-increasing real-valued function:

$$
\int_{a}^{b} g(c) F(c) d c \leq(\alpha-1) \int_{a}^{b} g(c) c f(c) d c
$$

Proof. Let $g(\cdot)$ be any non-increasing real-valued function and $F$ be a distribution, with density $f$, satisfying the condition in the proposition. Then, since $g^{\prime}(c) \leq 0$,

$$
\begin{aligned}
\int_{a}^{b} g(c)(F(c)-(\alpha-1) c f(c)) d c= & g(b) \int_{a}^{b}(F(s)-(\alpha-1) s f(s)) d s \\
& -\int_{a}^{b} g^{\prime}(c) \int_{a}^{c}(F(s)-(\alpha-1) s f(s)) d s d c
\end{aligned}
$$

is nonpositive if $\int_{a}^{y} F(c) d c \leq(\alpha-1) \int_{a}^{y} c f(c) d c$ holds for all $y \in[a, b]$. This latter condition is equivalent to $\mathbb{E}(X \mid X \leq y) \geq y / \alpha$, since integrating by parts

$$
\int_{a}^{y} F(c) d c-(\alpha-1) \int_{a}^{y} c f(c) d c=y F(y)-\alpha \int_{a}^{y} c f(c) d c
$$

which is nonpositive so long as $\mathbb{E}(X \mid X \leq y) \geq y / \alpha$.

3.1. Cost Loss Due to Lack of Information. We now turn compare the planner's expected cost when using the optimal mechanism to that in case she had complete information. From Proposition 13 , the former is given by:

$$
\mathcal{C}_{I}=\min _{x(c) \in \Gamma} \int_{c \in C}\left(\sum_{a \in A_{1}} x_{a}(c)\left[c_{a}+\frac{F_{a}\left(c_{a}\right)}{f_{a}\left(c_{a}\right)}\right]+\sum_{a \in A_{2}} x_{a}(c) c_{a}\right) f(c) d c .
$$

On the other hand, when complete information is available to the planner, her cost is:

$$
\mathcal{C}_{C}=\min _{x(c) \in \Gamma} \sum_{a \in A_{1}} \int_{c \in C} c_{a} x_{a}(c) f(c) d c+\sum_{a \in A_{2}} c_{a} \int_{c \in C} x_{a}(c) f(c) d c .
$$


Observe that if $A_{1}=A$, that is all costs are private information, and $F_{a}$ is uniform in $[0, s]$ for all $a \in A$, the planner's problem given by (4) is exactly the same as that in (5) with the costs doubled. Therefore the planner's expected cost in the optimal mechanism is twice as much as that in the complete information setting. In what follows we extend this result a very general class of distribution functions.

Proposition 5. If for all $a \in A_{1}$ the distribution $F_{a}$ satisfies that $\mathbb{E}(X \mid X \leq y) \geq y / \alpha$, where $X$ is drawn according to $F_{a}$, then $\mathcal{C}_{I} \leq \alpha \cdot \mathcal{C}_{C}$.

Proof. We apply lemma 4 to the planner's problem (5):

$$
\begin{aligned}
\mathcal{C}_{I} & \leq \min _{x(c) \in \Gamma, \nu_{a}\left(c_{a}\right) \downarrow} \int_{c \in C}\left(\sum_{a \in A_{1}} x_{a}(c) \alpha \cdot c_{a}+\sum_{a \in A_{2}} x_{a}(c) c_{a}\right) f(c) d c \\
& \leq \alpha \cdot \min _{x(c) \in \Gamma, \nu_{a}\left(c_{a}\right) \downarrow} \int_{c \in C}\left(\sum_{a \in A_{1}} x_{a}(c) c_{a}+\sum_{a \in A_{2}} x_{a}(c) c_{a}\right) f(c) d c \\
& \leq \alpha \cdot \mathcal{C}_{C},
\end{aligned}
$$

where the last inequality comes from relaxing the monotonicity constraint on $\nu_{a}$ and pointing out that the pointwise solution to the relaxed problem satisfies it automatically due to the monotonicity given by Lemma 1 .

Observation: The previous bound is related only to the distribution of private information about costs, and not to the particular problem $\Gamma$ being considered. As we already pointed out, in any instance of a combinatorial problem defined by $\Gamma$, when all resources are private and the information is distributed uniformly on $[0, a]$, this bound is tight.

Therefore we can apply such a result to any linear assignment problem and, as we discussed, this bound is tight when the distributions are uniform in $[0, s]$.

It is straightforward to observe that the the previous proposition can be applied to densities which are non-decreasing. Furthermore, as we show next, it can also be applied to an important class of distributions, namely, those that are symmetric and unimodal (SUD). A distribution function is unimodal if it has a unique local maximum.

Proposition 6. If $F_{a}$ is $S U D$ for all $a \in A_{1}$, then $\mathcal{C}_{I} \leq 2 \cdot \mathcal{C}_{C}$. Moreover, if $F_{a}$ is $S U D$ on $[r, s] \subset \mathbb{R}_{+}$ for all $a \in A_{1}$, then

$$
\mathcal{C}_{I} \leq \frac{2 s}{r+s} \cdot \mathcal{C}_{C}
$$

Proof. Let $F$ be SUD on $[r, s]$ and $X$ drawn according to $F$. Observe that, from Proposition 5, it is enough to show that $\mathbb{E}(X \mid X \leq y) \geq y / \alpha$, with $\alpha=2 s /(r+s)$. Observe that the latter condition is equivalent to

$$
h(y)=\int_{r}^{y}\left(x-\frac{y}{\alpha}\right) f(x) d x \geq 0 \quad \text { for all } y \in[r, s]
$$


This inequality clearly holds for $y=r$. Also, since $\mathbb{E}(X)=(s+r) / 2$, we have that $\int_{r}^{s} x f(x) d x=$ $(s+r) / 2=\int_{r}^{s} \frac{s}{\alpha} f(x) d x$, so the inequality also holds for $y=s$.

To prove that the inequality holds in general we can evaluate

$$
h^{\prime}(y)=\left(\frac{s-r}{2 s}\right) y f(y)-\left(\frac{s+r}{2 s}\right) \int_{r}^{y} f(x) d x .
$$

Note that $h^{\prime}(r) \geq 0$, and that for $y=r+\varepsilon$, with $\varepsilon$ smaller than $(s-r) / 2$, we have that

$$
\begin{aligned}
2 s \cdot h^{\prime}(y) & =(s-r)(r+\varepsilon) f(r+\varepsilon)-(s+r) \int_{r}^{r+\varepsilon} f(x) d x \\
& \geq(s-r)(r+\varepsilon) f(r+\varepsilon)-(s+r) \varepsilon f(r+\varepsilon) \\
& =f(r+\varepsilon) r(s-r-2 \varepsilon) \geq 0 .
\end{aligned}
$$

Furthermore, there exists an $\varepsilon \leq(s-r) / 2$ for which the first inequality is strict. We can conclude that $h^{\prime}(y) \geq 0$ for all $y \in[r,(r+s) / 2]$. We finish the proof by showing that $h^{\prime}(y)$ is nonincreasing for $y \in[(r+s) / 2, s]$, so that the sign of $h^{\prime}$ changes at most once in the interval $[r, s]$. Indeed, observe that $h^{\prime \prime}(y)=y f^{\prime}(y)(s-r) / 2 s-f(y) r / s$, and since $F$ is SUD, $f^{\prime}$ is nonpositive for $s \geq y \geq(r+s) / 2$.

The intuition behind the second part of this proposition is natural. For instance, if $r$ is very close to $s$, the cost under incomplete information approaches that of a fully informed planner. Also, if $s=r+K$, for constant $K$, the bound also goes to one as $r$ goes to infinity. This is because the amount of information the planner ignores is irrelevant when compared to the total cost of the project.

We finish this section by showing that one cannot expect to obtain a general bound for any class of distributions. Furthermore, even for decreasing distributions, the bound becomes arbitrarily bad. Indeed, consider the case where the planner must send one unit of flow from an origin to a destination, in a two link network. One of the links is private information with cost distribution proportional to $f(c)=1 /(c+\varepsilon)$ in $[0,1]$, while the other is public and its cost equals 1 (so $\Gamma=\{(x, y) \geq 0: x+y=1\})$. A simple calculation shows that

$$
\mathcal{C}_{I}>\frac{1}{2} \quad \text { and } \quad \mathcal{C}_{C}=\frac{1}{\ln (1+1 / \varepsilon)}-\varepsilon
$$

Thus, the ratio can be made arbitrarily large for small enough $\varepsilon$.

3.2. Cost loss due to efficiency. The standard VCG mechanism solves, for every cost realization $c$, the problem $\min \left\{c^{t} x \mid x \in \Gamma\right\}$ and assigns according to the solution rule $x_{a}^{V}(c)$ : It pays agent $a \in A_{1}, t_{a}(c)=c_{a} x_{a}(c)+\left(\sum_{b \in A} c_{b} x_{b}^{-a}(c)-\sum_{b \in A} c_{b} x_{b}(c)\right)$, where $x^{-a}(c)$ is a solution of $\min \left\{c^{t} x \mid x \in\right.$ $\left.\Gamma, x_{a}=0\right\}$. It is well known that such a mechanism is incentive compatible, but can involve infinite costs. However, if the support of the cost distribution is known, payments can be bounded without losing incentive compatibility. We denote such a mechanism, with payments given by $t_{a}(c)=\min \left\{c_{a} x_{a}(c)+\left(\sum_{b \in A} c_{b} x_{b}^{-a}(c)-\sum_{b \in A} c_{b} x_{b}(c)\right), \bar{c}_{a} x_{a}(c)\right\}$, the Truncated Vickrey-Clark-Groves (TVCG) mechanism. 
The TVCG mechanism assigns efficiently, but has a potentially higher cost than $\mathcal{C}_{I}$. However, the extra cost that TVCG incurs is bounded under suitable conditions on the distribution functions $F_{a}$, as we show in the next proposition.

Proposition 7. If for all $a \in A_{1}$ the distribution $F_{a}$ satisfies that $\mathbb{E}(X \mid X \leq y) \geq y / \alpha$, where $X$ is drawn according to $F_{a}$, then $\mathcal{C}_{V C G} \leq \alpha \cdot \mathcal{C}_{C} \leq \alpha \cdot \mathcal{C}_{I}$.

Proof. Since in the TVCG mechanism a resource owner with cost $\bar{c}_{a}$ obtains a net rent of 0 , we can write the total expected cost as

$$
\mathcal{C}_{V C G}=\int\left[\sum_{a \in A_{1}} x_{a}^{V}(c)\left[c_{a}+\frac{F_{a}\left(c_{a}\right)}{f_{a}\left(c_{a}\right)}\right]+\sum_{a \in A_{1}} x_{a}^{V}(c) c_{a}\right] f(c) d c
$$

where $x^{V}(c)$ corresponds to the efficient assignment given cost $c$, that is the solution to $\min \left\{c^{t} x \mid x \in\right.$ $\Gamma\}$ Therefore, using lemma 4, we can write

$$
\begin{aligned}
\mathcal{C}_{V C G} & =\int\left[\sum_{a \in A_{1}} x_{a}^{V}(c)\left[c_{a}+\frac{F_{a}\left(c_{a}\right)}{f_{a}\left(c_{a}\right)}\right]+\sum_{a \in A_{2}} x_{a}^{V}(c) c_{a}\right] f(c) d c \\
& \leq \alpha \int \sum_{a \in A} x_{a}^{V}(c) c_{a} f(c) d c \\
& =\alpha \mathcal{C}_{C} \leq \alpha \mathcal{C}_{I}
\end{aligned}
$$

Analogously to the previous section, we can use this result to find a tight bound when distributions are SUD.

Proposition 8. If $F_{a}$ is $S U D$ for all $a \in A_{1}$, then $\mathcal{C}_{V C G} \leq 2 \cdot \mathcal{C}_{C}$. Moreover, if $F_{a}$ is $S U D$ on $[r, s] \subset \mathbb{R}_{+}$for all $a \in A_{1}$, then

$$
\mathcal{C}_{V C G} \leq \frac{2 s}{r+s} \cdot \mathcal{C}_{C}
$$

Proof. Analogous to the proof of proposition 6.

With propositions 6 and 8 we have that, for SUD distributions in $[r, s]$ the following inequalities hold:

$$
\mathcal{C}_{C} \leq \mathcal{C}_{I} \leq \mathcal{C}_{V C G} \leq \frac{2 s}{r+s} \mathcal{C}_{C}
$$

A natural question is whether there is a better bound for the comparison between $\mathcal{C}_{I}$ and $\mathcal{C}_{V C G}$ than just $\mathcal{C}_{V C G} \leq \frac{2 s}{r+s} \cdot \mathcal{C}_{I}$. The answer is no, as sometimes the incomplete information planner has the same cost the fully informed planner, while the TVCG mechanism performs badly at a cost $\frac{2 s}{r+s} \cdot \mathcal{C}_{C}$. Consider for example the case where the planner must send one unit of flow between two nodes, in a two link network. One of the links is public while the other is private, i.e., $A_{1}=\left\{a_{1}\right\}$, $A_{2}=\left\{a_{2}\right\}$, and $\Gamma=\left\{\left(x_{a_{1}}, x_{a_{2}}\right) \geq 0: x_{a_{1}}+x_{a_{2}}=1\right\}$. Consider $\left\{F_{a_{1}}^{(n)}\right\}$ the family of SUDs for resource $a_{1}$, and assume that its support is the full interval $[0,1]$ and that $F_{a_{1}}^{(n)} \longrightarrow \delta_{1 / 2}$, where $\delta_{1 / 2}$ 
is the mass distribution putting probability 1 to $c_{a_{1}}=1 / 2$. Assume also that $c_{a_{2}}=1$. Then, we have that $\mathcal{C}_{V C G}^{(n)}=1$, but $\mathcal{C}_{I}^{(n)} \longrightarrow \mathcal{C}_{C} \equiv \frac{1}{2}$. Therefore our bound is tight.

\section{Computation and Implementation}

In general, implementing TVCG is no harder than solving $\left|A_{1}\right|$ times the original problem $\min \left\{c^{T} x: x \in \Gamma\right\}$, with the additional constraint that $x_{a}=0$. In some situations this latter problem can be solved even more efficiently [3]. The situation is different for optimal mechanisms. In fact, to implement an optimal mechanism the planner must compute the assignment and the payments only for a specific cost realization. Note that this is simpler than computing the whole assignment and payment rules, which require the assignment and payments for every cost realization.

Given a cost realization $c$, Proposition 13, states that the assignment can be computed as $\min \left\{c^{\prime T} x: x \in \Gamma\right\}$ for some virtual nonnegative cost vector $c^{\prime}$. This problem is the same as solving one instance of the complete information problem. However, to compute the payments $\bar{t}_{a}(c)=c_{a} \bar{x}_{a}(c)+\int_{c_{a}}^{\bar{c}_{a}} \bar{x}_{a}\left(t, c_{-a}\right) d t$ for a specific cost realization, in principle one needs to compute $\bar{x}_{a}\left(t, c_{-a}\right)$ for all $t \in\left[c_{a}, \bar{c}_{a}\right]$. That is we need to solve $\left|A_{1}\right|$ parametric optimization problems of the form:

$$
g_{i}(\theta)=\left(\arg \min _{x \in \Gamma}\left(c+\theta e_{i}\right)^{T} x\right)_{i},
$$

where $(\cdot)_{i}$ denotes the $i$-th component. The computational complexity of such a problem heavily depends on the structure of $\Gamma$ and determines the complexity of computing the optimal mechanism under incomplete information. In what follows we analyze three cases.

4.1. Case I: Parametric optimization is easy. If the parametric optimization problem (6) can be solved in polynomial time, then the whole mechanism can be computed in polynomial time as well. This includes the case in which $\Gamma$ is the set of all paths from a given source to a given sink, proved to be computationally easy in $[2,3]$.

Observe that a wider class of problem where parametric optimization turns out to be efficient is when $\Gamma=P \subseteq[0,1]^{|A|}$, with $P$ being an integral polytope. Of course a special case of this is $\Gamma=\{x: A x=\mathbb{1}, x \geq 0\}$, with $A$ totally unimodular. Shortest $s-t$ path is included in this class since it can be formulated imposing that the total flow across every $s-t$ cut equals one. Other problems in this class include minimum spanning tree and minimum perfect matching.

Proposition 9. If $\Gamma=P \subseteq[0,1]^{|A|}$, with $P$ an integral polytope, then (6) can be computed in polynomial time, by solving exactly two linear programming problems over $P$.

Proof. From Lemma1 $g_{i}(\cdot)$ is non-increasing. Also, since $\Gamma$ is a $\{0,1\}$ polytope we conclude that $g_{i}(\theta)$ is either 0 or 1 , for all $\theta \geq 0$. Therefore, to fully determine $g_{i}(\theta)$ (and thus obtain the optimal mechanism), it suffices to compute $\theta^{*}=\max \left\{\theta: g_{i}(\theta)=1\right\}$. 
To this end we first compute

$$
Z=\min \left\{\left(c+\theta e_{i}\right)^{T} x: x \in \Gamma, x_{i}=0\right\}=\min \left\{c_{-i}^{T} x_{-i}:\left(0, x_{-i}\right) \in \Gamma\right\} .
$$

Analogously we compute

$$
\theta+Z^{\prime}=\min \left\{\left(c+\theta e_{i}\right)^{T} x: x \in \Gamma, x_{i}=1\right\}=\theta+\min \left\{c_{-i}^{T} x_{-i}:\left(1, x_{-i}\right) \in \Gamma\right\} .
$$

Obtaining that $\theta^{*}=Z^{\prime}-Z$.

Remark that the previous proposition can easily be extended to the case in which $\Gamma$ is an integral polytope in $[0, K]^{|A|}$ for fixed $K$, or even for $K$ of polynomial size in the input. in this case we would need to solve $K$ linear programming problems over $\Gamma$. Furthermore, even for general $\Gamma$ but satisfying that the optimal solutions to $\min _{x \in \Gamma} c^{T} x$ lie in $\{0, \ldots, K\}^{|A|}$, the optimal mechanism can be obtained by solving $(K+1)\left|A_{1}\right|$ such problems.

4.2. Case II: Optimization is easy but parametric optimization is hard. Even if optimizing over $\Gamma$ is easy, the parametric optimization counterpart does not need to be so. For instance, for parametric linear programming, i.e., $\Gamma=\{A x=b, x \geq 0\}$, the function $g_{i}(\theta)$ can attain exponentially (in $|A|$ ) many different values [5]. Additionally, even for more structured problems such as minimum cost flow, $g_{i}(\theta)$ can have a superpolynomial number of values [1].

However, since resource owners are risk-neutral, we can easily obtain a randomized mechanism that is truthful and gives in expectation the same value, therefore it is also optimal.

Indeed, for a given a cost realization $c$, the assignment $\bar{x}(c)$ is computed exactly as before (i.e., by solving $\left.\min \left\{c^{T} x: x \in \Gamma\right\}\right)$, but the payments are computed using randomization. The payments to the owner of resource $a$, is given by $\bar{t}_{a}(c)=c_{a} \bar{x}_{a}(c)+\left(\bar{c}_{a}-c_{a}\right) \bar{x}_{a}\left(Y, c_{-a}\right)$, where $Y$ is a random variable uniformly distributed in $\left[c_{a}, \bar{c}_{a}\right]$. In expectation, which is all that matters to a risk-neutral resource owner, the latter payment equals

$$
c_{a} \bar{x}_{a}(c)+\left(\bar{c}_{a}-c_{a}\right) \int_{c_{a}}^{\bar{c}_{a}} \bar{x}_{a}\left(t, c_{-a}\right) \cdot \frac{1}{\bar{c}_{a}-c_{a}} d t=c_{a} \bar{x}_{a}(c)+\int_{c_{a}}^{\bar{c}_{a}} \bar{x}_{a}\left(t, c_{-a}\right) d t,
$$

and thus the mechanism is truthful and optimal. We conclude the following result.

Lemma 10. An optimal and truthful mechanism can be implemented by solving, for each $a \in A_{1}$, two problems of the form $\min \left\{c^{T} x: x \in \Gamma\right\}$.

Naturally the mechanism just described can be implemented in polynomial time so long as the optimization problem over $\Gamma$ can be solved in polynomial time. This enables us to implement a desirable mechanism even if the parametric optimization 6 is hard. However, this mechanism introduces high risk for the resource owners. To avoid this issue we could simply take a larger number $N$ of uniform samples $Y_{i}$ and compute $\bar{t}_{a}(c)=c_{a} \bar{x}_{a}(c)+\left(\bar{c}_{a}-c_{a}\right) \sum_{i=1}^{N} \bar{x}_{a}\left(Y_{i}, c_{-a}\right) / N$. With this the dispersion of payments will be reduced, though the computational effort will increase with $N$, leading to a tradeoff between risk and computational efficiency. 
4.3. Case III: Optimization is hard. We now study what happens when optimizing over $\Gamma$ is NP-hard, which is the case for a large number of combinatorial problems [8]. As one may expect, computing an optimal mechanism in such a case is also hard, so we can turn to search for truthful mechanism that are approximately optimal.

Suppose that we have an algorithm ALG for solving $\min _{x \in \Gamma} c^{T} x$, with an approximation guarantee of $\beta$. That is an algorithm returning a solution whose cost is at most $\beta$ times the optimal cost. Suppose furthermore that ALG is monotone, that is the returned solution $x_{a}^{\mathrm{ALG}}\left(c_{a}, c_{-a}\right)$ is decreasing in $c_{a}$. Then the mechanism that for each cost realization $c$ assigns according to $x^{\mathrm{ALG}}(c)$ is truthful. Moreover the expected cost for the planner of this mechanism is at most $\beta \cdot \mathcal{C}_{I}$.

Acknowledgments. The research of the first author was partially supported by CONICYT through grant Anillo en Redes ACT08. The research of the second author was partially supported by FONDECYT grant 11070159 and the Instituto Milenio en Sistemas Complejos de Ingeniería.

\section{REFERENCES}

[1] P. Carstensen. "Complexity of some parametric integer and network programming problems." Mathematical Programming 26(1):64-75, 1983.

[2] E. Elkind, A. Sahai, and K. Steiglitz. "Frugality in path auctions." Proceeding of the 15th ACM-SIAM Symposium on Discrete Algorithms (SODA), pp. 701-709, 2004.

[3] J. Hershberger and S. Suri. "Vickrey prices and shortest paths: What is an edge worth?" Proceeding of the 42nd IEEE Symposium on Foundations of Computer Science (FOCS), pp. 252-259, 2001.

[4] V. Krishna and E. Maenner. "Convex potentials with an application to mechanism design." Econometrica 69(4):1113-1119, 2001.

[5] K. Murty. "Computational complexity of parametric linear programming." Mathematical Programming 19:213219, 1980.

[6] R. Myerson. "Optimal auction design." Mathematics of Operations Research 6(1):58-73, 1981.

[7] N. Nissan, T. Roughgarden, E. Tardos, and V. Vazirani. "Algorithmic Game Theory." Cambridge University Press, New York, 2007.

[8] A. Schrijver. "Combinatorial Optimization: Polyhedra and Efficiency." Springer-Verlag, Berlin, 2003. 


\section{Appendix A. The Optimal Bayesian Mechanism}

Recall that $V_{a}\left(c_{a}\right)=\max _{c_{a}^{\prime} \in C_{a}} U_{a}\left(c_{a}, c_{a}^{\prime}\right)$, and that $U_{a}\left(c_{a}, c_{a}^{\prime}\right)$ is linear in $c_{a}$. Then $V_{a}\left(c_{a}\right)$ is a maximum of linear functions, and therefore convex and therefore differentiable almost everywhere. This allows a simple characterization of incentive compatibility. For $a \in A_{1}$ let the expected utilization of resource be,

$$
\nu_{a}\left(c_{a}\right):=\int_{C_{-a}} x_{a}\left(c_{a}, c_{-a}\right) f_{-a}\left(c_{-a}\right) d c_{-a} .
$$

Lemma 11. A mechanism satisfies (IC) if and only if:

(i) $\nu_{a}\left(c_{a}\right)$ is non-increasing (i.e., weakly decreasing).

(ii) $V_{a}\left(c_{a}\right)=V_{a}\left(\bar{c}_{a}\right)+\int_{c_{a}}^{\bar{c}_{a}} \nu_{a}(s) d s$

Proof. Note that a mechanism satisfies (IC) if and only if $\max _{c_{a}^{\prime} \in C_{a}} U_{a}\left(c_{a}, c_{a}^{\prime}\right)$ is attained at $c_{a}^{\prime}=c_{a}$, which in turn is equivalent to $-\nu_{a}\left(c_{a}\right) \in \partial V_{a}\left(c_{a}\right)$, where $\partial V_{a}\left(c_{a}\right)$ is the subgradient of $V_{a}$ at $c_{a}$. With this, we can easily check the two implications needed. First, note that since $V_{a}\left(c_{a}\right)$ is convex, a selection from its subgradient must be monotone, then $-\nu_{a}\left(c_{a}\right)$ is nondecreasing. On the other hand, a result in [4] implies that a generalization of the fundamental theorem of calculus holds for convex functions, therefore (ii) is satisfied since $-\nu_{a}\left(c_{a}\right) \in \partial V_{a}\left(c_{a}\right)$.

To prove the other implication observe that from (ii) and (i) we can conclude that

$$
V_{a}\left(c_{a}^{\prime}\right)-V_{a}\left(c_{a}\right)=\int_{c_{a}^{\prime}}^{c_{a}} \nu_{a}(s) d s \geq\left(c_{a}-c_{a}^{\prime}\right) \cdot \nu_{a}\left(c_{a}\right)=\left(c_{a}^{\prime}-c_{a}\right) \cdot\left(-\nu_{a}\left(c_{a}\right)\right) .
$$

Therefore $-\nu_{a}\left(c_{a}\right) \in \partial V_{a}\left(c_{a}\right)$ and the mechanism is incentive compatible.

The previous lemma establishes that the assignment rules determine the owners' payoffs up to a constant. Therefore they determine the payment rules up to a constant. Moreover, it allows for a simple characterization of the participation constraint (PC) whenever a mechanism is (IC).

Corollary 12. A mechanism that satisfies (IC), also satisfies (PC), if and only if $V_{a}\left(\bar{c}_{a}\right) \geq 0$.

We now rewrite the objective function of the planner.

$$
\int_{c \in C}\left(\sum_{a \in A_{1}} t_{a}(c)+\sum_{a \in A_{2}} c_{a} x_{a}(c)\right) f(c) d c=\sum_{a \in A_{1}} \int_{c \in C} t_{a}(c) f(c) d c+\int_{c \in C} \sum_{a \in A_{2}} c_{a} x_{a}(c) f(c) d c
$$

From the definition of $V_{a}(\cdot)$ and Lemma 11, the first term can be evaluated as

$$
\begin{aligned}
& \sum_{a \in A_{1}} \int_{\underline{c}_{a}}^{\bar{c}_{a}} V_{a}\left(\bar{c}_{a}\right) f_{a}\left(c_{a}\right) d c_{a}+\int_{\underline{c}_{a}}^{\bar{c}_{a}} \int_{c_{a}}^{\bar{c}_{a}} \nu_{a}(y) d y f_{a}\left(c_{a}\right) d c_{a}+\sum_{a \in A_{1}} \int_{C} c_{a} x_{a}(c) f(c) d c \\
= & \sum_{a \in A_{1}} V_{a}\left(\bar{c}_{a}\right)+\int_{\underline{c}_{a}}^{\bar{c}_{a}} \nu_{a}(y) \int_{\underline{c}_{a}}^{y} f_{a}\left(c_{a}\right) d c_{a} d y+\sum_{a \in A_{1}} \int_{C} c_{a} x_{a}(c) f(c) d c \\
= & \sum_{a \in A_{1}} V_{a}\left(\bar{c}_{a}\right)+\int_{\underline{c}_{a}}^{\bar{c}_{a}} \int_{C_{-a}} x_{a}\left(y, c_{-a}\right) f_{-a}\left(c_{-a}\right) d c_{-a} F_{a}(y) d y+\sum_{a \in A_{1}} \int_{C} c_{a} x_{a}(c) f(c) d c \\
= & \sum_{a \in A_{1}} V_{a}\left(\bar{c}_{a}\right)+\int_{c \in C} \sum_{a \in A_{1}} x_{a}(c)\left[c_{a}+\frac{F_{a}\left(c_{a}\right)}{f_{a}\left(c_{a}\right)}\right] f(c) d c .
\end{aligned}
$$


We conclude that the planners' objective function can be written as a function of the assignment rules only plus a term which, as we will see later, vanishes in an optimal mechanism.

$$
\int_{c \in C}\left(\sum_{a \in A_{1}} x_{a}(c)\left[c_{a}+\frac{F_{a}\left(c_{a}\right)}{f_{a}\left(c_{a}\right)}\right]+\sum_{a \in A_{2}} x_{a}(c) c_{a}\right) f(c) d c+\sum_{a \in A_{1}} V_{a}\left(\bar{c}_{a}\right) .
$$

From Lemma 11, Corollary 12, and from the objective function (7), we are ready to write the planners' problem as a function of the assignment rules and characterize the optimal mechanism.

Proposition 13. The optimal assignment rules $\bar{x}(c)=\left\{\bar{x}_{a}(c)\right\}_{a \in A}$ are those solving, for each cost revelations $\left\{c_{a}\right\}_{a \in A_{1}}$, the following optimization problem:

$$
\min _{y \in \Gamma} \sum_{a \in A_{1}}\left(c_{a}+\frac{F_{a}\left(c_{a}\right)}{f_{a}\left(c_{a}\right)}\right) y_{a}+\sum_{a \in A_{2}} c_{a} y_{a}
$$

and an optimal payment rule is given by $\bar{t}_{a}(c)=c_{a} \bar{x}_{a}(c)+\int_{c_{a}}^{\bar{c}_{a}} \bar{x}_{a}\left(t, c_{-a}\right) d t$. In other words, $x(c)$ is the minimum cost assignment in $\Gamma$ with virtual costs $c_{a}^{\prime}=c_{a}+F_{a}\left(c_{a}\right) / f_{a}\left(c_{a}\right)$ for all $a \in A_{1}$, and $c_{a}^{\prime}=c_{a}$ for all $a \in A_{2}$.

Proof. First note that an optimal mechanism satisfies $V_{a}\left(\bar{c}_{a}\right)=0$. Then, the last term in the planner's objective (7) vanishes. Let us now show that if $x(c) \in \Gamma$ and $\nu_{a}\left(c_{a}\right)$ is non-increasing, then it satisfies (IC) and $(\mathrm{PC})$, so long as the payments are $t_{a}(c)=c_{a} x_{a}(c)+\int_{c_{a}}^{\bar{c}_{a}} x_{a}\left(s, c_{-a}\right) d s$. Indeed, observe that $V_{a}\left(c_{a}\right)=$ $\int_{C_{-a}} t_{a}\left(c_{a}, c_{-a}\right) f_{-a}\left(c_{-a}\right) d c_{-a}-\int_{C_{-a}} c_{a} x_{a}\left(c_{a}, c_{-a}\right) f_{-a}\left(c_{-a}\right) d c_{-a}$. Replacing the value of the payments we obtain

$$
V_{a}\left(c_{a}\right)=\int_{C_{-a}} \int_{c_{a}}^{\bar{c}_{a}} x_{a}\left(t, c_{-a}\right) d t f_{-a}\left(c_{-a}\right) d c_{-a}=\int_{c_{a}}^{\bar{c}_{a}} \nu_{a}(t) d t
$$

implying that $x(c)$ satisfies (IC) and (PC) with $V_{a}\left(\bar{c}_{a}\right)=0$. Then the assignment rule $x(c)$ is a solution to the optimal control problem

$$
\begin{array}{ll}
\min _{\left\{x_{a}(c)\right\}_{a \in A}} & \int_{c \in C}\left(\sum_{a \in A_{1}} x_{a}(c)\left[c_{a}+\frac{F_{a}\left(c_{a}\right)}{f_{a}\left(c_{a}\right)}\right]+\sum_{a \in A_{2}} x_{a}(c) c_{a}\right) f(c) d c \\
\text { s.t. } & x(c) \in \Gamma \text { and } \nu_{a}\left(c_{a}\right) \text { non-increasing for all } a \in A_{1} .
\end{array}
$$

Notice that $\bar{x}(c)$, as defined in the statement of the proposition, solves previous problem pointwise if we relax the constraint asking for $\nu_{a}\left(c_{a}\right)$ being non-increasing. Furthermore, since $c_{a}+F_{a}\left(c_{a}\right) / f_{a}\left(c_{a}\right)$ is nondecreasing, Lemma 1 implies that $\bar{v}_{a}\left(\cdot, c_{-a}\right)$ is non-increasing. With this, $\nu_{a}\left(c_{a}\right)=\int_{C_{-a}} \bar{x}_{a}\left(c_{a}, c_{-a}\right) d c_{-a}$, is non-increasing as well. 